\title{
Management and obstetric prognosis of pregnancy with isolated cutaneous lupus
}

\section{Aida Oulehri, Hanane Baybay, Soukaina Chhiti, Sara Elloudi, Zakia Douhi, Fatima Zahra Mernissi}

Department of Dermatology, University Hospital Hassan II, Fez, Morocco

Corresponding author: Dr. Aida Oulehri, E-mail: aidaoulehri@gmail.com

\begin{abstract}
It is well known that pregnancy in a woman with systemic lupus erythematosus (SLE) is associated with an increased risk of adverse maternal and fetal outcomes. But what about the risk in patients with isolated cutaneous lupus erythematosus (CLE) during pregnancy? There are insufficient data on clinical obstetrical and neonatal outcomes in women with CLE and very few studies have been published concerning the obstetric prognosis in the various types of cutaneous lupus. We report the case of a 24-year-old woman with no known pathological history, who presented with a severe and inaugural flare-up of a typical acute lupus erythematosus (ACLE) without systemic involvement which was complicated by a fetal loss.
\end{abstract}

Key words: Cutaneous lupus erythematosus; Obstetric prognosis; Neonatal outcomes; Management

\section{INTRODUCTION}

Lupus erythematosus (LE) is included among the so called connective tissue diseases and is divided into one systemic form - SLE and one cutaneous form - CLE. They can occur both together and separately [1]. SLE is a systemic autoimmune disease that primarily affects women of childbearing age. This chronic disease is distinguished by its multiorgan involvement, characteristic inflammatory lesions of the skin, joints, serous membranes, kidneys and central nervous system, and its association with high titers of autoantibodies to an array of autoantigens [2]. The various cutaneous manifestations of $\mathrm{LE}$ are divided into LE-nonspecific and LE-specific skin disease based on histologic criteria. LE-specific skin disease includes the subtypes of CLE such as acute cutaneous LE (ACLE), subacute cutaneous LE (SCLE), chronic cutaneous LE (CCLE), and intermittent CLE (ICLE). The subdivision of these subtypes with different prognosis and course is supported by genetic, clinical, histologic, and immunoserologic findings. The subtypes of CLE require a specific morphological and clinical analysis [3]. The LEnonspecific skin manifestations include a wide range of symptoms with different histopathological pictures such as vascular skin lesions. The LEnonspecifc skin manifestations are not exclusive to LE disease but are often seen in patients with active SLE but also in several other autoimmune diseases [1]. Pregnancy in a woman with SLE is well known associated with an increased risk of adverse maternal and fetal outcomes, flares of disease activity, preeclampsia, fetal loss, intrauterine growth retardation and preterm birth are established risks of such pregnancies. The purpose of our work is to raise the problem of the very little known and little studied obstetrical and neonatal prognosis of pregnant women with isolated cutaneous lupus without systemic disease, which is just as much a pregnancy at risk as illustrated by the outcome of fetal loss in our patient. We also discuss the therapeutic modalities that can be offered to these patients during their pregnancies.

\section{CASE REPORT}

The patient is a 24-year-old woman with no notable pathological history, who presented an acute and

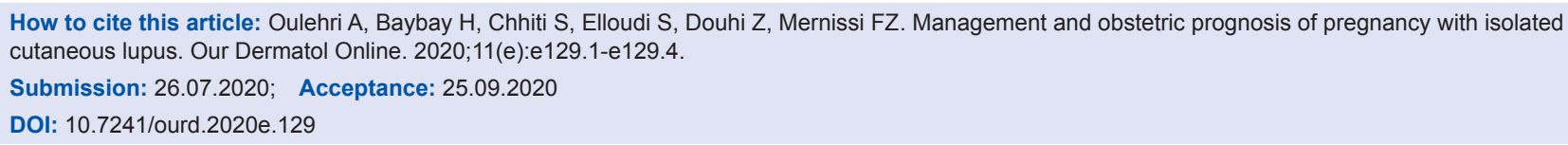


diffuse rash after one month of pregnancy. The rash started on his face two weeks before his consultation. The patient had no previous sun exposure and was not taking any specific medication. On her face she had an erythematous erosive butterfly rash typically over both cheeks, extends over the nasal bridge, and spares the nasolabial folds (Fig. la). The eruption of the body shows an erythematous and erosive maculopapular none pruritic, exanthema distributed symmetrically, involving the neckline, the upper back (Fig. 1b), the backs of the hands and fingers with involving the interphalangeal areas (Fig. 1c), and the roots of the limbs, with erosions of the oral and genital mucosa and several partially alopecic plaques of the scalp (Fig. 1d). The rest of the general examination showed no abnormalities. There were no systemic signs on interrogation, anti-nuclear antibodies, native DNA and SSA were positive, the rest of the immunological work-up was negative. The diagnosis of acute and generalized cutaneous lupus erythematosus has been made; a full work-up for systemic damage was negative. The patient was put on hydroxychloroquine; the evolution was marked by fetal loss, followed by the complete resolution of the cutaneous episode.

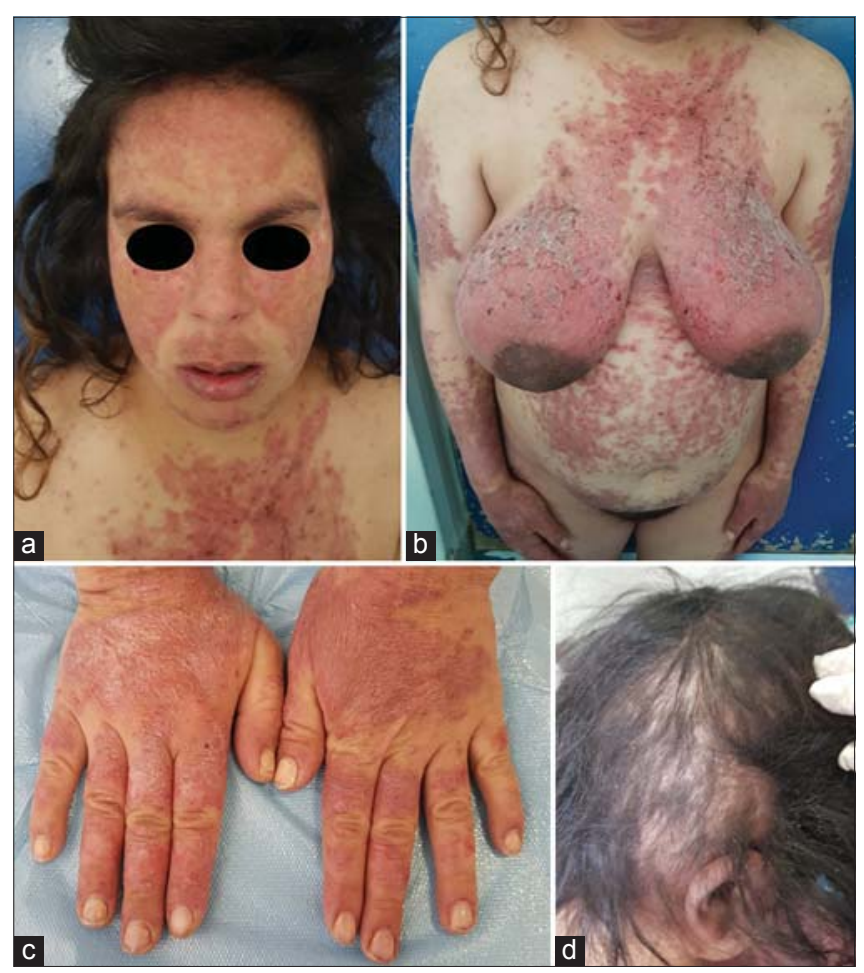

Figure 1: Clinical images at patient admission: (a) butterfly-like appearance, (b) extensive rash, (c) typical involvement of the backs of the hands in the interphalangeal areas, (d) alopecic plaques.

\section{DISCUSSION}

CLE is two to three times more frequent than SLE. Similar to proposed etiologies for SLE, current theories discuss a multifactorial relationship leading to the development of cutaneous lupus, including genetic susceptibility, auto-immune induction and immunesystem damage [4]. Cutaneous manifestations are very common in SLE patients; over $80 \%$ display skin symptoms sometime during the course of the disease and in $20-25 \%$ of patients cutaneous manifestations are the first symptom of SLE disease [5]. ACLE can occur as localized or generalized disease. The more common localized form is characterized by "butterfly erythema" which usually spreads symmetrically over the bridge of the nose and the cheeks, typically sparing the nasolabial folds; the sharply bordered erythema is frequently mistaken by patients for sunburn. Smaller erythematous lesions can initially appear which later merge and develop into papules and plaques, in addition, severe edema, scaling, erosions, and crusts may occur.

Generalized ACLE usually presents with morbilliform or maculopapular, sometimes pruritic, exanthema, consisting of multiple, erythematous confluent macules and papules. The rash spreads out symmetrically over the entire body, often also involving the backs of the hands and extensor surfaces of the fingers. On the distal phalanges of the fingers and toes there is patchy or diffuse erythema, sometimes with small hemorrhagic areas [3]. Acute SLE is almost always associated with systemic disease and the most typical patient is a young woman in her twenties to thirties with fair skin [6].

Usually, the generalized form of ACLE is less common and is associated with increased disease activity of SLE. Histologically, ACLE lesions show liquefactive degeneration of the basal layer, oedema of the upper dermis and a scattered interface, perivascular and periadnexal lymphocytic infiltrate, all of which are generally less pronounced as compared to other CLE sub-types. Immunologically, a positive ANA is found in $95 \%$ of ACLE patients, as well as a high incidence of anti-double-stranded DNA (anti-DNA) and anti-Sm antibodies [5].

Pregnancy in a woman with SLE is associated with an increased risk of adverse maternal and fetal outcomes, flares of disease activity, preeclampsia, fetal loss, intrauterine growth retardation and preterm birth 
are established risks of such pregnancies. Patients should delay conception until their lupus has been in remission for at least 6 months. The most common organs affected in lupus flares during pregnancy are skin, kidney, blood and joints [2]. The majority of cases are known lupus patients who have flare-ups during pregnancy; the occurrence of newly diagnosed lupus in a patient with no known history of lupus, as in the case of our patient is much rarer.

A very few studies have been published concerning the obstetric prognosis in the various types of cutaneous lupus. One cohort study [7] compared the obstetric prognosis of women presenting isolated signs of cutaneous lupus (butterfly rash, discoid lupus, subacute lupus erythematosus, nasal or oral ulcers: group 1), SLE (group 2) and group 3 (controls). They conclude that CLE has comparable pregnancy outcomes to those seen in healthy women. Lower rates of disease exacerbation and anti-phospholipid antibodies are conducive to a better pregnancy outcome in cutaneous lupus compared with organ-affecting systemic disease. In the same study the rates of pregnancy loss in women with SLE and CLE was respectively 35.8 and $13.4 \%$ [7]; which shows that foetal loss is certainly less frequent in isolated CLE but remains a described complication that should be expected, as our case shows.

The presence in pregnant women with CLE of laboratory signs of antiphospholipid syndrome must be taken into consideration and suitable monitoring is recommended [8].

Anti-SSA antibodies are found in $72 \%$ of patients presenting subacute lupus erythematosus, $47 \%$ of patients presenting acute lupus erythematosus, and $22 \%$ of patients presenting chronic lupus erythematosus. Anti-SSB antibodies were found respectively in 36\%, $27 \%$ and $7 \%$ of patients [9]. Maternal anti-SSA/Ro and anti-SSB/La antibodies cross the placental barrier in the same way as all $\operatorname{IgG}$ and may cause neonatal lupus in the fetus or newborn [10]. Close ultrasound monitoring has been recommended for patient with isolated CLE in the presence of anti-SSA or anti-SSB antibodies [8].

Topical corticosteroids may be continued regardless of the trimester of pregnancy, preferably in a preparation containing no other active substances. During breastfeeding, prolonged contact must be avoided between the baby and the area of skin being treated. Plasma concentrations of tacrolimus applied to skin are low and systemic adverse effects of this drug are rare at the usual dosages. Its use is authorized during pregnancy and breastfeeding [11]. The use of dapsone is possible at any term of pregnancy, at the lowest effective dose, the amount of dapsone ingested via milk is significant; the baby receives up to $19 \%$ of the maternal dose (in $\mathrm{mg} / \mathrm{kg}$ ). In view of this, it is preferable to avoid breast-feeding when taking dapsone. Thalidomide is strictly contraindicated and retinoids are not authorized during pregnancy. The data available in pregnant women exposed to hydroxychloroquine during pregnancy are very numerous and reassuring, its use in pregnant women is recommended by many international scientific societies [11].

\section{CONCLUSION}

The prognosis, the various complications, the monitoring modalities and the management of pregnancies associated with systemic lupus are well codified and known to all. On the other hand, very few data exist for pregnancies with isolated cutaneous lupus without systemic organ damage. However, this is indeed can be a high-risk pregnancy, as our case illustrates.

Large studies of pregnancy outcomes in patients followed for isolated cutaneous lupus erythematosus would be very useful to better determine the obstetrical prognosis of these patients and to better monitor and manage them during pregnancy.

\section{Consent}

The examination of the patient was conducted according to the Declaration of Helsinki principles. The authors certify that they have obtained all appropriate patient consent forms.

\section{REFERENCES}

1. Grönhagen CM, Nyberg F. Cutaneous lupus erythematosus: An update. Indian Dermatol Online J. 2014;5:8.

2. Stojan G, Baer AN. Flares of systemic lupus erythematosus during pregnancy and the puerperium: prevention, diagnosis and management. Expert Rev Clin Immunol. 2012;8:439-53.

3. Kuhn A, Sticherling M, Bonsmann G. Clinical manifestations of cutaneous lupus erythematosus. J Dtsch Dermatol Ges. 2007;5:1124-37.

4. Okon LG, Werth VP. Cutaneous Lupus Erythematosus: Diagnosis and treatment. Best Pract Res Clin Rheumatol. 2013;27:391-404.

5. Rothfield N, Sontheimer RD, Bernstein M. Lupus erythematosus: systemic and cutaneous manifestations. Clin Dermatol. 2006;24:348-62.

6. Parodi A, Cozzani E. Cutaneous manifestations of lupus erythematosus. G Ital Dermatol Venereol. 2014;149:549-54.

7. Hamed HO, Ahmed SR, Alzolibani A, Kamal MM, Mostafa MS, 


\section{www.odermatol.com}

Gamal RM, et al. Does cutaneous lupus erythematosus have more favorable pregnancy outcomes than systemic disease? A two-center study. Acta Obstet Gynecol Scand. 2013;92:934-42.

8. Guettrot-Imbert G, Morel N, Le Guern V, Plu-Bureau G, Frances C, Costedoat-Chalumeau N. Pregnancy and contraception in systemic and cutaneous lupus erythematosus. Ann Dermatol Venereol. 2016;143):590-600.

9. Biazar C, Sigges J, Patsinakidis N, Ruland V, Amler S, Bonsmann G, et al. Cutaneous lupus erythematosus: first multicenter database analysis of 1002 patients from the European Society of Cutaneous Lupus Erythematosus (EUSCLE). Autoimmun Rev. 2013;12:444-54.
10. Morel N, Georgin-Lavialle S, Levesque K, Guettrot-Imbert G, Le Guern V, Le Bidois J, et al. [Neonatal lupus syndrome: Literature review]. Rev Med Interne. 2015;36:159-66.

11. http://www.lecrat.org/

Copyright by Aida Oulehri, et al. This is an open-access article distributed under the terms of the Creative Commons Attribution License, which permits unrestricted use, distribution, and reproduction in any medium, provided the original author and source are credited.

Source of Support: Nil, Conflict of Interest: None declared. 